International Journal of Applied Mathematical Research, 1 (4) (2012) 433-451

(C) Science Publishing Corporation

www.sciencepubco.com/index.php/IJAMR

\title{
Investigating the Propagation and Death of Information in Human Subpopulation Networks
}

\author{
Jomar F. Rabajante \\ Institute of Mathematical Sciences and Physics, \\ University of the Philippines Los Baños \\ Email: jfrabajante@up.edu.ph
}

\begin{abstract}
We introduce a conceptual-mathematical model that simulates the spread of information. We represent the states of the actors in the information flow using compartments. The compartment diagram is translated into a system of coupled ordinary differential equations. The diversity of social groups (subpopulations) is incorporated in the model, and the model is applicable for a heterogeneous community. We determine four possible spots that can be influenced to control the information propagation such as (1) controlling the inflow and outflow of people in each subpopulation, (2) regulating the media of communication, (3) influencing the belief system of the actors, and (4) introducing an antithesis to the circulating information.
\end{abstract}

Mathematics Subject Classification: 91D99

Keywords: compartment model, correcting rumors, dynamical systems, information propagation, social contagion

\section{Introduction}

Social interaction is part of the ecology of humans. Communication is one of the ways species interact. In humans, communication involves transfer of information. Studying information propagation may help create decisions such as in controlling organizational grapevines, minimizing damages caused by rumors, regulating spread of wrong information during disasters, and disseminating important news. 
The speed of information propagation depends on the significance of the information to the receivers as well as on the level of awareness of the members of the community (such as in the case of advertised information). The truthfulness of the information based on evidences, the trustworthiness of the spreader of information, the need of any information (whether true or false) just to answer anxieties, and the degree of suggestibility and credulity of the people often influence information spread. The paper [7] discusses how rumors, gossips and urban legends are transmitted.

Various social scientists have already formulated theories about information propagation, and some of these theories are based on intuition and qualitative studies. However, experimental research is impractical and usually infeasible to validate such theories. The application of mathematics, especially dynamical systems, is one of the strategies in investigating social phenomenon - not to replace experimental validation but to observe more the implications of the existing theories and conjectures. Mathematical and computational simulations are commonly used to investigate in a conceptual and theoretical level the complex interactions during information flow.

Rumor spread is one of the common social phenomena that researchers study. Rosnow and Foster [11] explain the basic principles behind rumors and gossips. Various models were formulated to study rumor propagation, such as the Daley-Kendall and Maki-Thompson models which are inspired by the S-I$\mathrm{R}$ (Susceptible-Infected-Removed) disease epidemics model [1, 2, 3, 6, 9]. Serge Galam, the father of sociophysics, as well as Kawachi analyze the dynamics of rumor spread using mathematics [4, 5, 8]. Umali and Rabajante [14], Salvania and Pabico [12], Suo and Chen [13], and Nekovee et al. [10] investigate the spread of information, such as gossips, in complex social networks. The study of information propagation is also useful in analyzing the behavior of financial markets [15].

We propose a new model that considers a community with different subpopulations where people have a choice to believe the shared information. This model also incorporates the possibility of introducing an information that opposes the circulating information.

In our model we consider a community of humans. We call the members of the population as actors, which are likewise members of subpopulations. A subpopulation can represent a clique in the social network or group of people in a specific geographic location. We assume that the subpopulations are mutually disjoint, that is, no member of one subpopulation is a member of the other.

We also consider the closeness, nature of fidelity and belief systems of each subpopulations. The interaction between two subpopulations may be more frequent than the interaction with the other subpopulations. Moreover, some subpopulations may consider the information from one subpopulation more 
believable than the information from the other.

We refer to the actors who have not yet heard the circulating information as ignorants, actors who have believed the circulating information as believers, and believers that spread the information as spreaders. We represented and visualized the information propagation process using a compartment diagram, and the compartments in the diagram represent the states of the actors.

\section{Assumptions and Compartment Diagram}

Our model illustrates the behavior of transferring information from spreaders to other members of the community and the change in the states of the actors. The model is based on the classical I-S-S (Ignorant-Spreader-Stifler) rumor spreading model. The assumptions of the model are as follows:

- The model is deterministic and continuous in state space and time.

- There are only two circulated information that are unaltered during the process. We focus on one main information, and the other information is a total negation (opposite) of the main information. We refer to the opposite information as the antithesis.

- The subpopulations (groups, cliques, factions or clusters) are mutually disjoint; and no member can directly transfer to another group during the time frame of the simulation.

- Each subpopulation is homogeneous, that is, the members of a subpopulation share the same characteristics (e.g., ability to interact, fidelity, credulity, suggestibility).

- Rates of flow from one state to another follow the conservation law, that is, all actors in the model are accounted.

- All new members of the community are ignorants.

- Per unit of time, a person can only listen to a single spreader, but a spreader can transfer information to several people simultaneously (such as in the case of broadcast information). A spreader is always active in sharing the information.

- Believers of the main information do not spread the antithesis as well as non-believers do not spread the main information.

- A person decides to believe or not to believe the information immediately once the information is conveyed. 
Let us define the following sets and parameters for $i=1,2, \ldots, n$ and $j=$ $1,2, \ldots, n$, where $n$ is the number of mutually disjoint subpopulations in the community (note that $P_{i}, P_{i} C S, P_{i} B$ and $P_{i} N B$ compose the subpopulation $i)$ :

$P_{i}$ : the set of ignorants in subpopulation $i$;

$P_{i} C S$ : the set of ignorants in subpopulation $i$ having contact with spreaders of main information;

$P_{i} B$ : the set of individuals in subpopulation $i$ believing the main information;

$P_{i} N B$ : the set of individuals in subpopulation $i$ not believing the main information (non-believers);

$a_{i}$ : the constant average number of new members of subpopulation $i$ per unit of time $t$;

$b_{i}$ : the expected proportion of $P_{i}$ exiting the community per unit of time;

$c_{i}$ : the expected proportion of $P_{i}$ having contact with spreaders of main information from all subpopulations per unit of time;

$\operatorname{prob}_{i j}$ : the probability that a spreader of main information from subpopulation $j$ will have a contact with an actor from subpopulation $i$ per unit of time;

$q{ } b_{i j}$ : the probability that a spreader of antithesis from subpopulation $j$ will have a contact with a believer in $P_{i} B$ per unit of time;

$S_{j i}$ : the average proportion of $P_{j} B$ that are spreaders of main information who can interact with actors from subpopulation $i$ at time $t$;

$Z_{j i}$ : the average proportion of $P_{j} N B$ that are spreaders of antithesis who can interact with believers from $P_{i} B$ at time $t$;

$L_{i j}$ : the average number of members of subpopulation $i$ that a spreader of main information from subpopulation $j$ can interact with per unit of time;

$V_{i j}$ : the average number of members of subpopulation $i$ that a spreader of antithesis from subpopulation $j$ can interact with per unit of time;

$d_{i}$ : the probability that a person from $P_{i} C S$ will believe the conveyed main information (or the expected proportion of $P_{i} C S$ that will believe the main information per unit of time); 
$e_{i}$ : the probability that a person from $P_{i} C S$ will not believe the conveyed main information (or the expected proportion of $P_{i} C S$ that will not believe the main information per unit of time). Note that $d_{i}+e_{i}=1$;

$p b_{i}$ : the probability that a believer in $P_{i} B$ who had an interaction with a spreader of antithesis will believe the antithesis (or the expected proportion of $P_{i} B$ who had an interaction with a spreader of antithesis that will believe the antithesis per unit of time);

$f_{i}$ : the expected proportion of $P_{i} B$ exiting the community per unit of time;

$g_{i}$ : the expected proportion of $P_{i} N B$ exiting the community per unit of time;

$h_{i}$ : the expected proportion of $P_{i} B$ who listen and believe the spreaders of antithesis from all subpopulations per unit of time; and

$k_{i}$ : the expected proportion of $P_{i} N B$ having contact with spreaders of main information from all subpopulations per unit of time.

Let $[\cdot]$ denote the cardinality of the set. We represent the states of the actors in the information flow using compartments. The compartment model of our information propagation model where the focus is the main information (not the antithesis) is shown in Figure 1.

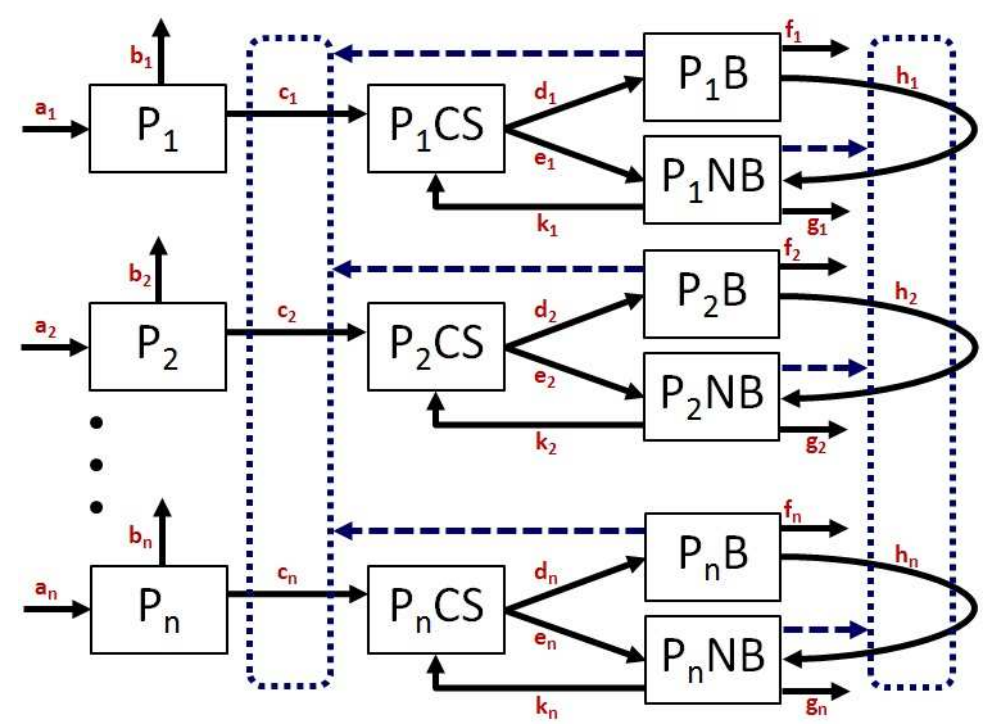

Figure 1: An Information Propagation Compartment Model. Solid lines denote flow of actors from one state to another while dashed lines denote influence.

There are actors who enter and exit $P_{i}$, and some members of $P_{i}$ move to $P_{i} C S$. Actors that transfer from $P_{i}$ to $P_{i} C S$ are those who hear the main 
information shared by the spreaders. The set of spreaders of main information is a subset of the set of believers.

Actors from $P_{i} C S$ move to either $P_{i} B$ or $P_{i} N B$. Actors who believe the main information transfer to $P_{i} B$, or otherwise to $P_{i} N B$.

There is a probability that a non-believer in $P_{i} N B$ may believe the main information after interacting with a spreader. However, there is also a chance that a believer in $P_{i} B$ may change his belief and transfer to $P_{i} N B$ after interacting with a spreader of the antithesis. The set of spreaders of antithesis is a subset of the set of non-believers.

Spreaders of the main information or of the antithesis can influence actors in another subpopulation, e.g., a spreader from $P_{1} B$ can interact and share the main information to an ignorant in $P_{2}$. If $\left[P_{i} B\right]=0$ for all $i$ then it means the main information is no longer in circulation and we call this as "dead" information.

The formula for $c_{i}$ is as follows:

$$
\begin{aligned}
c_{i} & =\frac{\sum_{j=1}^{n}\left(\text { prob }_{i j}\right)\left(S_{j i}\right)\left(\left[P_{j} B\right]\right)\left(L_{i j}\right)}{\left[P_{i}\right]} \text { if }\left[P_{i}\right] \neq 0 \\
c_{i} & =0 \text { if }\left[P_{i}\right]=0 \\
i & =1,2, \ldots, n .
\end{aligned}
$$

The contact rate $c_{i}$ and $k_{i}$ are proportional to the number of believers present since we define $S_{j}$ as the average proportion of $P_{j} B$ that are spreaders of main information at time $t . L_{i j}$ can be interpreted as the broadcasting capacity of a spreader of main information, e.g., if $L_{12}=4$ then, on the average, a spreader of main information from subpopulation 2 can talk to four ignorants in subpopulation 1.

The product $\left(\operatorname{prob}_{i j}\right)\left(S_{j i}\right)\left(\left[P_{j} B\right]\right)\left(L_{i j}\right)$ represents the number of ignorants in subpopulation $i$ having interaction with spreaders of main information from subpopulation $j$. Since we assume that an actor listens to at most one spreader at time $t$ then $W_{i j_{1}} \cap W_{i j_{2}}=\emptyset$ for all $i, j_{1} \neq j_{2}$ (where $W_{i j}$ denotes the set of ignorants in subpopulation $i$ having interaction with spreaders of main information from subpopulation $j$ at time $t$ ). It follows that the total number of ignorants in subpopulation $i$ having interaction with spreaders of main information from all subpopulations is $\sum_{j=1}^{n}\left(\operatorname{prob}_{i j}\right)\left(S_{j i}\right)\left(\left[P_{j} B\right]\right)\left(L_{i j}\right)$.

Likewise, the formula for $k_{i}$ and $h_{i}$ are

$$
k_{i}=\frac{\sum_{j=1}^{n}\left(\operatorname{prob}_{i j}\right)\left(S_{j i}\right)\left(\left[P_{j} B\right]\right)\left(L_{i j}\right)}{\left[P_{i} N B\right]} \text { if }\left[P_{i} N B\right] \neq 0
$$




$$
\begin{aligned}
k_{i} & =0 \text { if }\left[P_{i} N B\right]=0 \\
i & =1,2, \ldots, n, \text { and } \\
h_{i} & =\frac{\sum_{j=1}^{n}\left(q r o b_{i j}\right)\left(Z_{j i}\right)\left(\left[P_{j} N B\right]\right)\left(V_{i j}\right)\left(p b_{i}\right)}{\left[P_{i} B\right]} \text { if }\left[P_{i} B\right] \neq 0 \\
h_{i} & =0 \text { if }\left[P_{i} B\right]=0 \\
i & =1,2, \ldots, n .
\end{aligned}
$$

The rate at which an ignorant becomes a believer is $d_{i} c_{i}$ and the rate at which a non-believer becomes a believer is $d_{i} k_{i}$. Moreover, the rate at which an individual exits $P_{i} B$ is $f_{i}+h_{i}$. Hence, the basic reproductive rate of the spread of the main information is $\frac{d_{i} c_{i}+d_{i} k_{i}}{f_{i}+h_{i}}$. It should be noted that the parameters $c_{i}$, $k_{i}$ and $h_{i}$ should not be greater than 1 .

\section{ODE Model and Control Spots}

We translate the compartment diagram into an autonomous system of coupled ordinary differential equations (ODEs) with $\left[P_{i}\right],\left[P_{i} B\right]$ and $\left[P_{i} N B\right], i=$ $1,2, \ldots, n$ as the state variables. The actors in $P_{i} C S, i=1,2, \ldots, n$ are in transient states, and we presume that a person decides to believe or not to believe the information immediately once the information is conveyed (i.e., $\left[P_{i} C S\right]$ quickly vanishes at each time $\left.t\right)$. Thus, for simplicity, we disregard $P_{i} C S$ in the diagram.

The system of ordinary differential equations that corresponds to our simplified compartment model is

$$
\begin{aligned}
\frac{d\left[P_{i}\right]}{d t} & =a_{i}-\left(b_{i}+c_{i}\right)\left[P_{i}\right] \\
\frac{d\left[P_{i} B\right]}{d t} & =d_{i}\left(c_{i}\left[P_{i}\right]+k_{i}\left[P_{i} N B\right]\right)-\left(f_{i}+h_{i}\right)\left[P_{i} B\right] \\
\frac{d\left[P_{i} N B\right]}{d t} & =e_{i}\left(c_{i}\left[P_{i}\right]+k_{i}\left[P_{i} N B\right]\right)-\left(g_{i}+k_{i}\right)\left[P_{i} N B\right]+h_{i}\left[P_{i} B\right] \\
i & =1,2, \ldots, n .
\end{aligned}
$$

Substituting the formula of $c_{i}, k_{i}$ and $h_{i}$, we now have the following piecewise (conditional) linear system of ODEs:

$$
\frac{d\left[P_{i}\right]}{d t}=a_{i}-b_{i}\left[P_{i}\right]+\sum_{j=1}^{n}\left(\operatorname{prob}_{i j}\right)\left(S_{j i}\right)\left(\left[P_{j} B\right]\right)\left(L_{i j}\right),\left[P_{i}\right] \neq 0
$$




$$
\begin{aligned}
& \frac{d\left[P_{i}\right]}{d t}=a_{i},\left[P_{i}\right]=0 \\
& \frac{d\left[P_{i} B\right]}{d t}=2 d_{i} \sum_{j=1}^{n}\left(\operatorname{prob}_{i j}\right)\left(S_{j i}\right)\left(\left[P_{j} B\right]\right)\left(L_{i j}\right)-f_{i}\left[P_{i} B\right] \\
& -\sum_{j=1}^{n}\left(q r o b_{i j}\right)\left(Z_{j i}\right)\left(\left[P_{j} N B\right]\right)\left(V_{i j}\right)\left(p b_{i}\right), \\
& {\left[P_{i}\right] \neq 0,\left[P_{i} B\right] \neq 0,\left[P_{i} N B\right] \neq 0} \\
& \frac{d\left[P_{i} B\right]}{d t}=d_{i} \sum_{j=1}^{n}\left(\operatorname{prob}_{i j}\right)\left(S_{j i}\right)\left(\left[P_{j} B\right]\right)\left(L_{i j}\right)-f_{i}\left[P_{i} B\right] \\
& -\sum_{j=1}^{n}\left(q r o b_{i j}\right)\left(Z_{j i}\right)\left(\left[P_{j} N B\right]\right)\left(V_{i j}\right)\left(p b_{i}\right), \\
& {\left[P_{i}\right]=0,\left[P_{i} B\right] \neq 0,\left[P_{i} N B\right] \neq 0} \\
& \frac{d\left[P_{i} B\right]}{d t}=d_{i} \sum_{j=1}^{n}\left(\operatorname{prob}_{i j}\right)\left(S_{j i}\right)\left(\left[P_{j} B\right]\right)\left(L_{i j}\right) \\
& -\sum_{j=1}^{n}\left(q r o b_{i j}\right)\left(Z_{j i}\right)\left(\left[P_{j} N B\right]\right)\left(V_{i j}\right)\left(p b_{i}\right), \\
& {\left[P_{i}\right] \neq 0,\left[P_{i} B\right]=0,\left[P_{i} N B\right] \neq 0} \\
& \frac{d\left[P_{i} B\right]}{d t}=2 d_{i} \sum_{j=1}^{n}\left(\operatorname{prob}_{i j}\right)\left(S_{j i}\right)\left(\left[P_{j} B\right]\right)\left(L_{i j}\right)-f_{i}\left[P_{i} B\right], \\
& {\left[P_{i}\right] \neq 0,\left[P_{i} B\right] \neq 0,\left[P_{i} N B\right]=0} \\
& \frac{d\left[P_{i} B\right]}{d t}=-\sum_{j=1}^{n}\left(q r o b_{i j}\right)\left(Z_{j i}\right)\left(\left[P_{j} N B\right]\right)\left(V_{i j}\right)\left(p b_{i}\right), \\
& {\left[P_{i}\right]=0,\left[P_{i} B\right]=0,\left[P_{i} N B\right] \neq 0} \\
& \frac{d\left[P_{i} B\right]}{d t}=d_{i} \sum_{j=1}^{n}\left(\operatorname{prob}_{i j}\right)\left(S_{j i}\right)\left(\left[P_{j} B\right]\right)\left(L_{i j}\right)-f_{i}\left[P_{i} B\right], \\
& {\left[P_{i}\right]=0,\left[P_{i} B\right] \neq 0,\left[P_{i} N B\right]=0} \\
& \frac{d\left[P_{i} B\right]}{d t}=d_{i} \sum_{j=1}^{n}\left(\text { prob }_{i j}\right)\left(S_{j i}\right)\left(\left[P_{j} B\right]\right)\left(L_{i j}\right), \\
& {\left[P_{i}\right] \neq 0,\left[P_{i} B\right]=0,\left[P_{i} N B\right]=0} \\
& \frac{d\left[P_{i} B\right]}{d t}=0,\left[P_{i}\right]=0,\left[P_{i} B\right]=0,\left[P_{i} N B\right]=0 \\
& \frac{d\left[P_{i} N B\right]}{d t}=2 e_{i} \sum_{j=1}^{n}\left(\operatorname{prob}_{i j}\right)\left(S_{j i}\right)\left(\left[P_{j} B\right]\right)\left(L_{i j}\right)-g_{i}\left[P_{i} N B\right] \\
& -\sum_{j=1}^{n}\left(\operatorname{prob}_{i j}\right)\left(S_{j i}\right)\left(\left[P_{j} B\right]\right)\left(L_{i j}\right)
\end{aligned}
$$




$$
\begin{aligned}
& +\sum_{j=1}^{n}\left(q r o b_{i j}\right)\left(Z_{j i}\right)\left(\left[P_{j} N B\right]\right)\left(V_{i j}\right)\left(p b_{i}\right), \\
& {\left[P_{i}\right] \neq 0,\left[P_{i} B\right] \neq 0,\left[P_{i} N B\right] \neq 0} \\
& \frac{d\left[P_{i} N B\right]}{d t}=e_{i} \sum_{j=1}^{n}\left(\operatorname{prob}_{i j}\right)\left(S_{j i}\right)\left(\left[P_{j} B\right]\right)\left(L_{i j}\right)-g_{i}\left[P_{i} N B\right] \\
& -\sum_{j=1}^{n}\left(\operatorname{prob}_{i j}\right)\left(S_{j i}\right)\left(\left[P_{j} B\right]\right)\left(L_{i j}\right) \\
& +\sum_{j=1}^{n}\left(q r o b_{i j}\right)\left(Z_{j i}\right)\left(\left[P_{j} N B\right]\right)\left(V_{i j}\right)\left(p b_{i}\right), \\
& {\left[P_{i}\right]=0,\left[P_{i} B\right] \neq 0,\left[P_{i} N B\right] \neq 0} \\
& \frac{d\left[P_{i} N B\right]}{d t}=2 e_{i} \sum_{j=1}^{n}\left(\operatorname{prob}_{i j}\right)\left(S_{j i}\right)\left(\left[P_{j} B\right]\right)\left(L_{i j}\right)-g_{i}\left[P_{i} N B\right] \\
& -\sum_{j=1}^{n}\left(\operatorname{prob}_{i j}\right)\left(S_{j i}\right)\left(\left[P_{j} B\right]\right)\left(L_{i j}\right) \\
& {\left[P_{i}\right] \neq 0,\left[P_{i} B\right]=0,\left[P_{i} N B\right] \neq 0} \\
& \frac{d\left[P_{i} N B\right]}{d t}=e_{i} \sum_{j=1}^{n}\left(\operatorname{prob}_{i j}\right)\left(S_{j i}\right)\left(\left[P_{j} B\right]\right)\left(L_{i j}\right) \\
& +\sum_{j=1}^{n}\left(q r o b_{i j}\right)\left(Z_{j i}\right)\left(\left[P_{j} N B\right]\right)\left(V_{i j}\right)\left(p b_{i}\right), \\
& {\left[P_{i}\right] \neq 0,\left[P_{i} B\right] \neq 0,\left[P_{i} N B\right]=0} \\
& \frac{d\left[P_{i} N B\right]}{d t}=e_{i} \sum_{j=1}^{n}\left(\operatorname{prob}_{i j}\right)\left(S_{j i}\right)\left(\left[P_{j} B\right]\right)\left(L_{i j}\right)-g_{i}\left[P_{i} N B\right] \\
& -\sum_{j=1}^{n}\left(\operatorname{prob}_{i j}\right)\left(S_{j i}\right)\left(\left[P_{j} B\right]\right)\left(L_{i j}\right) \\
& {\left[P_{i}\right]=0,\left[P_{i} B\right]=0,\left[P_{i} N B\right] \neq 0} \\
& \frac{d\left[P_{i} N B\right]}{d t}=\sum_{j=1}^{n}\left(q r o b_{i j}\right)\left(Z_{j i}\right)\left(\left[P_{j} N B\right]\right)\left(V_{i j}\right)\left(p b_{i}\right), \\
& {\left[P_{i}\right]=0,\left[P_{i} B\right] \neq 0,\left[P_{i} N B\right]=0} \\
& \frac{d\left[P_{i} N B\right]}{d t}=e_{i} \sum_{j=1}^{n}\left(\operatorname{prob}_{i j}\right)\left(S_{j i}\right)\left(\left[P_{j} B\right]\right)\left(L_{i j}\right) \\
& {\left[P_{i}\right] \neq 0,\left[P_{i} B\right]=0,\left[P_{i} N B\right]=0} \\
& \frac{d\left[P_{i} N B\right]}{d t}=0,\left[P_{i}\right]=0,\left[P_{i} B\right]=0,\left[P_{i} N B\right]=0 \\
& i=1,2, \ldots, n \text {. }
\end{aligned}
$$

If $a_{i}=0$ for all $i$, then we will have a non-homogeneous system of ODEs. 
However, we can redefine $a_{i}$ to be proportional to $\left[P_{i}\right]+\left[P_{i} B\right]+\left[P_{i} N B\right]$, that is, the number of new members entering the subpopulation is proportional to the number of actors in the subpopulation. On the other hand, we can redefine $b_{i}\left[P_{i}\right], f_{i}\left[P_{i} B\right]$ and $g_{i}\left[P_{i} N B\right]$ to be constants.

We classify the parameters into four modules - the inflow and outflow module, the communication (for the main information) module, the belief module, and the counterattack module. See Figure 2 for the illustration. The following is the list of the parameters belonging to each module:

- Inflow and outflow module: $a_{i}, b_{i}, f_{i}$ and $g_{i}$

- Communication module: $c_{i}$ and $k_{i}$

- Belief module: $d_{i}$ and $e_{i}$

- Counterattack module: $h_{i}$

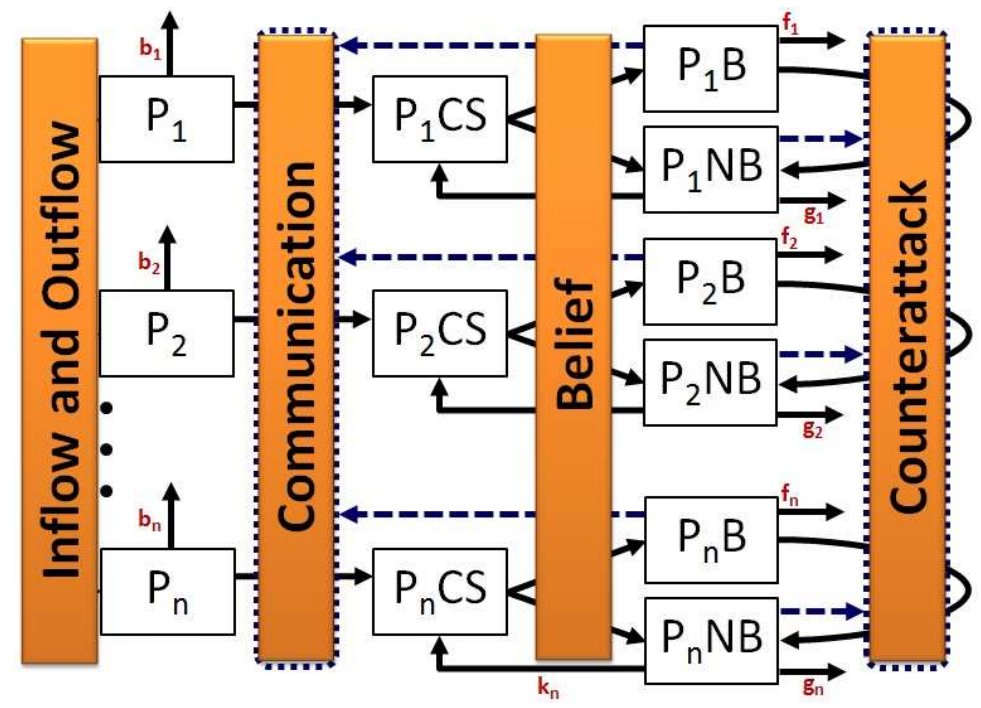

Figure 2: Possible Information Spread Control Spots.

These four modules are control spots that can be influenced to regulate the propagation of the main information. The inflow and outflow module describes the migration of actors in the community. As more new members continually enter the community through $P_{i}$, the longer the main information may circulate because $P_{i}$ is the source of possible new believers. However, it is usually infeasible or impractical to control the migration of people inside a community.

If we increase $c_{i}$ and $k_{i}$ in the communication module, more actors will hear the main information and thus, the larger the chance $\left[P_{i} B\right]$ will increase. 
Effective advertising and communication media can increase the value of $c_{i}$ and $k_{i}$. On the other hand, in controlling rumor spread, inhibiting communication among actors (e.g., decreasing the value of $c_{i}$ and $k_{i}$ ) may result to a bigger problem. Absence of communication drives the actors to create other stories to answer their curiosities and anxieties.

If we influence the belief module, such as decreasing $d_{i}$ and increasing $e_{i}$, then we can regulate the flow of people to $P_{i} B$ and $P_{i} N B$. However, it is very difficult to change the belief of people unless effective (usually costly) advertising campaigns and debates are done.

One strategy to decrease the number of believers is to introduce an antithesis to the main information. This strategy entails increased effort in broadcasting the antithesis and convincing the believers. In our model, increasing the number of non-believers may result to an effective antithesis campaign. The Conflict Research Consortium, University of Colorado [16] suggested strategies to control rumors, and one of these strategies is the introduction of an antithesis:

"The key to effective rumor control efforts is an ability to perform three functions. First, some mechanism is needed for determining what rumors are actually circulating. Second, an effective strategy is needed for determining which rumors are true, and which are false. Finally, mechanisms are needed for correcting inaccurate rumors and replacing them with reliable information."

\subsection{Simulation}

Consider two subpopulations $(n=2)$. We numerically solved the system (Equations 7 to 23) for a given set of parameters. Figures 6 to 15 show some simulations in time series where the value of a specific parameter is varied.

Some of our numerical simulations (see Figure 3 for an illustration) show that the time series of $\left[P_{i} B\right]$ fluctuates at an early period and levels (usually approaching an equilibria) at a later period.

In some cases, the parameters $a_{i}$ and $b_{i}$ in the inflow and outflow module do not significantly affect $\left[P_{i} B\right]$ and $\left[P_{i} N B\right]$ unlike the other parameters. Based on our system (Equations 7 to 23 ), increasing $a_{i}$ and decreasing $b_{i}$ do not necessarily increase the number of believers and non-believers because the parameters $a_{i}$ and $b_{i}$ do not appear in $\frac{d\left[P_{i} B\right]}{d t}$ and $\frac{d\left[P_{i} N B\right]}{d t}$. However, $a_{i}$ and $b_{i}$ affect $\left[P_{i}\right]$ and as we increase $a_{i}$ and decrease $b_{i}$, the number of ignorants increases. As the number of ignorants increases, the life span of the main information may increase since $P_{i}$ is the source of possible new believers and spreaders. The effect of the inflow of new members is significantly noticeable when there is minimal number of actors exiting the community (outflow). As 


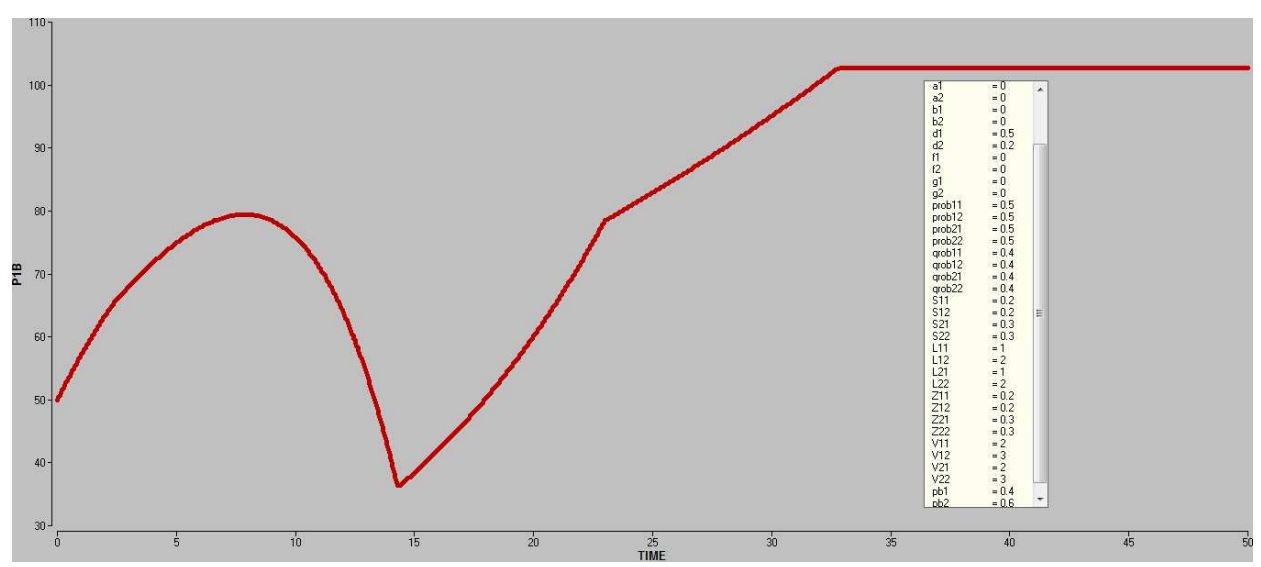

Figure 3: An example where the time series of $\left[P_{i} B\right]$ fluctuates at an early period and levels at a later period.

shown in Figure 4, the change in the value of $a_{i}$ may induce variation in the behavior of $\left[P_{i} B\right]$ especially when there is inhibited outflow.

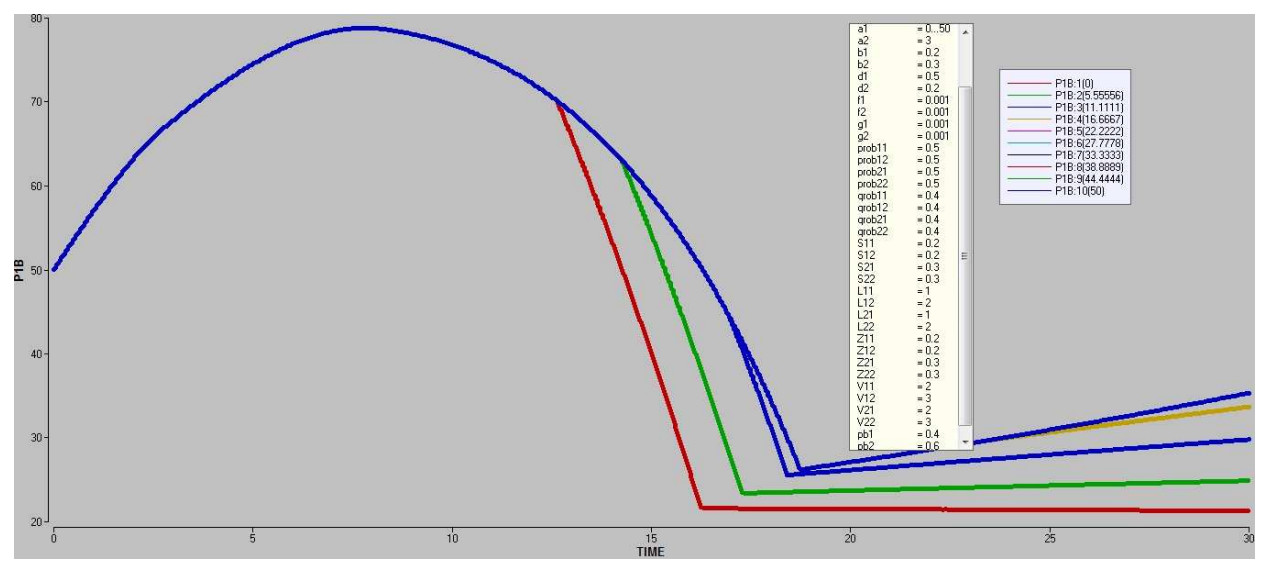

Figure 4: An instance when $a_{i}$ affects $\left[P_{i} B\right]$.

In our system (Equations 7 to 23) where the number of ignorants is large, the propagation of the main information does not significantly depend on the number of ignorants but on the number of spreaders and their ability to disseminate the information. It also depends on the chance that actors would listen and believe the spreaders. However, if we use another formula for $c_{i}$ (e.g., $c_{i}$ is equal to a positive constant) then we can make the number of ignorants matter. In reality, sometimes the number of ignorants may or may not matter in information propagation.

We investigate another case where we assumed $c_{i}, k_{i}$ and $h_{i}$ are constants. This assumption is only valid when $\left[P_{i} B\right]$ is always nonzero if $c_{i}>0$ or $k_{i}>0$ because if $\left[P_{i} B\right]=0$ then there will be no spreader of main information. 
Solving the equilibrium points $\left(\left[P_{i}\right]^{*},\left[P_{i} B\right]^{*},\left[P_{i} N B\right]^{*}\right)$ of our system (Equations 4 to 6 ) leads to $\left[P_{i}\right]^{*}=\frac{a_{i}}{b_{i}+c_{i}}$ where either $b_{i}$ or $c_{i}$ is nonzero. It follows that

$$
\begin{aligned}
\frac{d_{i} c_{i} a_{i}}{b_{i}+c_{i}}+d_{i} k_{i}\left[P_{i} N B\right]^{*}-\left(f_{i}+h_{i}\right)\left[P_{i} B\right]^{*} & =0 \\
\frac{e_{i} c_{i} a_{i}}{b_{i}+c_{i}}+\left(e_{i} k_{i}-g_{i}-k_{i}\right)\left[P_{i} N B\right]^{*}+h_{i}\left[P_{i} B\right]^{*} & =0 .
\end{aligned}
$$

which imply that

$$
\begin{aligned}
{\left[P_{i} B\right]^{*} } & =\frac{\frac{d_{i} c_{i} a_{i}}{b_{i}+c_{i}}+d_{i} k_{i}\left[P_{i} N B\right]^{*}}{f_{i}+h_{i}} \\
{\left[P_{i} N B\right]^{*} } & =\frac{\frac{e_{i} c_{i} a_{i}}{b_{i}+c_{i}}\left(f_{i}+h_{i}\right)+h_{i} \frac{d_{i} c_{i} a_{i}}{b_{i}+c_{i}}}{-\left(\left(e_{i} k_{i}-g_{i}-k_{i}\right)\left(f_{i}+h_{i}\right)+h_{i} d_{i} k_{i}\right)} .
\end{aligned}
$$

Notice that it is necessary for either $f_{i}$ or $h_{i}$ to be nonzero for the above $\left[P_{i} B\right]^{*}$ to be defined. It can easily be seen that if either $b_{i}=c_{i}=0$ or $f_{i}=h_{i}=0$ then no equilibrium exists (assuming $a_{i}>0, c_{i}>0$ and $d_{i}>0$ ). Moreover, $\left(e_{i} k_{i}-g_{i}-k_{i}\right)\left(f_{i}+h_{i}\right)+h_{i} d_{i} k_{i}<0$ for $\left[P_{i} N B\right]^{*}$ to be nonnegative and defined.

From our analytic investigation we can observe that all parameters affect the behavior of our system. Notice that $\left[P_{i} B\right]^{*}$ and $\left[P_{i} N B\right]^{*}$ are in terms of all the parameters. However, note that $\left(\left[P_{i}\right]^{*},\left[P_{i} B\right]^{*},\left[P_{i} N B\right]^{*}\right)$ may be stable or unstable given some parameter values.

The representative Jacobian of the system (Equations 4, 5 and 6) where $c_{i}$, $k_{i}$ and $h_{i}$ are constants is as follows:

$$
\left[\begin{array}{ccc}
-\left(b_{i}+c_{i}\right) & 0 & 0 \\
d_{i} c_{i} & -\left(f_{i}+h_{i}\right) & d_{i} k_{i} \\
e_{i} c_{i} & h_{i} & e_{i} k_{i}-g_{i}-k_{i}
\end{array}\right]
$$

There are three eigenvalues of the above Jacobian matrix which will give us an idea if $\left(\left[P_{i}\right]^{*},\left[P_{i} B\right]^{*},\left[P_{i} N B\right]^{*}\right)$ is stable. If the real part of the eigenvalues are all negative then the equilibrium point is stable. The eigenvalues are

$$
\begin{aligned}
& \lambda_{1}=-b_{i}-c_{i} \\
& \lambda_{2}=-\frac{1}{2} g_{i}-\frac{1}{2} h_{i}-\frac{1}{2} k_{i}-\frac{1}{2} f_{i}+\frac{1}{2} k_{i} e_{i}+\frac{1}{2} A \\
& \lambda_{3}=-\frac{1}{2} g_{i}-\frac{1}{2} h_{i}-\frac{1}{2} k_{i}-\frac{1}{2} f_{i}+\frac{1}{2} k_{i} e_{i}-\frac{1}{2} A
\end{aligned}
$$

where 


$$
\begin{aligned}
A= & \left(k_{i}^{2} e_{i}^{2}-2 k_{i}^{2} e_{i}-2 f_{i} g_{i}+2 f_{i} h_{i}-2 g_{i} h_{i}-2 f_{i} k_{i}+2 g_{i} k_{i}-2 h_{i} k_{i}+f_{i}^{2}\right. \\
& \left.+g_{i}^{2}+h_{i}^{2}+k_{i}^{2}+4 d_{i} h_{i} k_{i}+2 f_{i} k_{i} e_{i}-2 g_{i} k_{i} e_{i}+2 h_{i} k_{i} e_{i}\right)^{\frac{1}{2}}
\end{aligned}
$$

We can explore other possible formulas for computing $c_{i}$, such as hyperbolic and sigmoidal (Hill) equations. However, if we use a hyperbolic or a sigmoidal equation then our system (Equations 4, 5 and 6) will be nonlinear. A possible formula for $c_{i}$ is

$$
c_{i}=\frac{\left[P_{i}\right]+\sum_{j=1}^{n} S_{j i}\left[P_{j} B\right]}{1+\left[P_{i}\right]+\sum_{j=1}^{n} S_{j i}\left[P_{j} B\right]} .
$$

Notice that the above formula incorporates the effect of the number of ignorants and spreaders in communicating the main information. Similar formula can also be used for $k_{i}$ and $h_{i}$. A sample simulation is shown in Figure 5 where $c_{i}, k_{i}$ and $h_{i}$ are of hyperbolic-type.

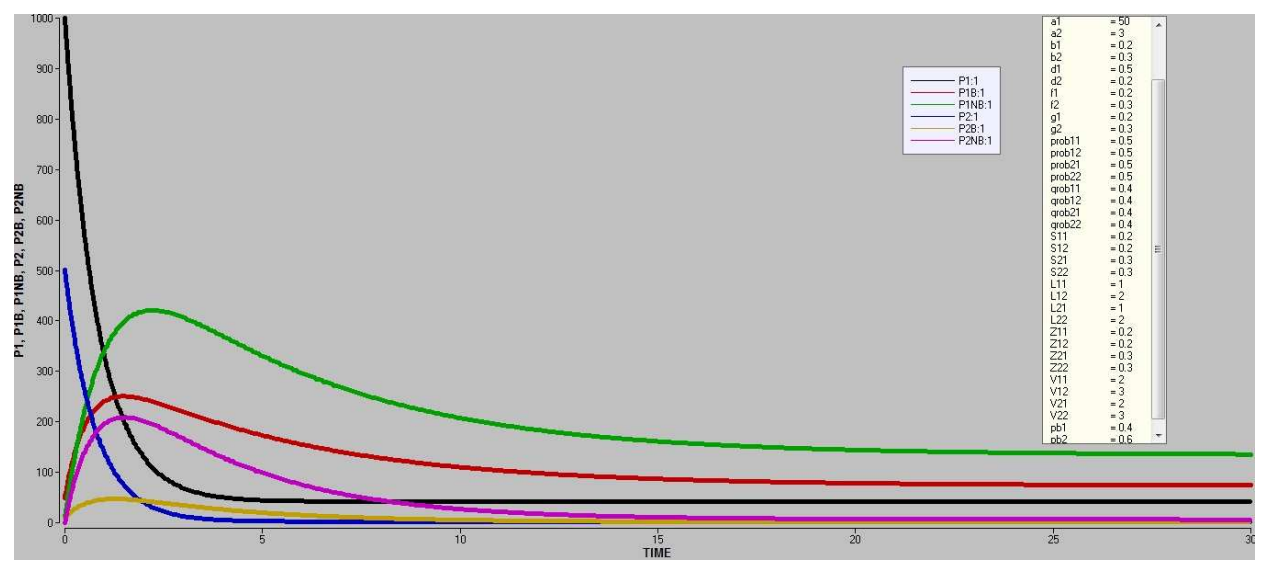

Figure 5: An example simulation where $c_{i}, k_{i}$ and $h_{i}$ are of hyperbolic-type.

\section{Concluding Remarks}

Even without performing an actual experiment to study information propagation, theoretical and conceptual modeling help us understand the general behavior of the information propagation process. In this research, we determined four possible control spots to regulate information spread - the migration of 
actors, the interaction with spreaders, the degree of skepticism of receivers, and the introduction of an opposite information. However, one of the difficult tasks in using theoretical models is the determination of the actual values of the parameters and input values. Furthermore, we did not include the effect of time delays and presence of stochastic noise. We recommend to extend our mathematical model to include more social factors, such as presence of multiple information, to further understand the propagation and death of information.

\section{References}

[1] D.J. Daley, D.G. Kendall, "Epidemics and Rumours", Nature, Vol.204, (1964), p.1118.

[2] D.J. Daley, D.G. Kendall, "Stochastic Rumours", IMA Journal of Applied Mathematics, Vol.1, No.1, (1965), pp.42-55.

[3] P.S. Dodds, D.J. Watts, "A generalized model of social and biological contagion", Journal of Theoretical Biology, Vol.232, (2005), pp.587-604.

[4] S. Galam, "Modelling rumors: the no plane Pentagon French hoax case", Physica A, Vol.320, (2003), pp.571-580.

[5] S. Galam, "Heterogeneous beliefs, segregation, and extremism in the making of public opinions", Physical Review E, Vol.71, (2005), doi:10.1103/PhysRevE.71.046123.

[6] W. Goffman, V.A. Newill, "Generalization of Epidemic Theory: An Application to the Transmission of Ideas", Nature, Vol.204, (1964), pp.225-228.

[7] B. Guerin, Y. Miyazaki, " Analyzing Rumors, Gossips, and Urban Legends Through Their Conversational Properties", The Psychological Record, Vol.56, No.1, (2006), url: http://opensiuc.lib.siu.edu/tpr/vol56/iss1/2.

[8] K. Kawachi et al., "A rumor transmission model with various contact interactions", Journal of Theoretical Biology, Vol.253, (2008), p.5560.

[9] D.P. Maki, M. Thompson, Mathematical models and applications, with emphasis on the social, life, and management sciences, Prentice Hall, (1973).

[10] M. Nekovee et al., "Theory of Rumour Spreading in Complex Social Networks", Physica A, Vol.374, (2007), pp.457-470. 
[11] R.L. Rosnow, E.K. Foster, "Rumor and Gossip Research", Psychological Science Agenda, Vol.19, No.4, (2005), url: http://www.apa.org/science/about/psa/2005/04/gossip.aspx.

[12] A.C. Salvania, J.P. Pabico, "Information Spread Over an Internetmediated Social Network: Phases, Speed, Width, and Effects of Promotion", Philippine Information Technology Journal, Vol.3, No.2, (2010), pp.15-25.

[13] S. Suo, Y. Chen, "The Dynamics of Public Opinion in Complex Networks", Journal of Artificial Societies and Social Communication, Vol.11, No.42, (2008), url: http://jasss.soc.surrey.ac.uk/11/4/2.html.

[14] R.E.D.C. Umali, J.F. Rabajante, "A Mathematical Model of Rumor Propagation for Disaster Management", Journal of Nature Studies, Vol.10, No.2, (2011), pp.61-70.

[15] S. Wang, C. Zhang, "Microscopic model of financial markets based on belief propagation", Physica A, Vol.354, (2005), pp.496504.

[16] Conflict Research Consortium, University of Colorado. Rumor Control Teams. url: http://www.colorado.edu/conflict/peace/treatment/rumorct.htm, accessed October 9, 2011.

[17] Software used for simulation: Berkeley Madonna (ver. 8.3.18). R.I. Macey, G.F. Oster, University of California.

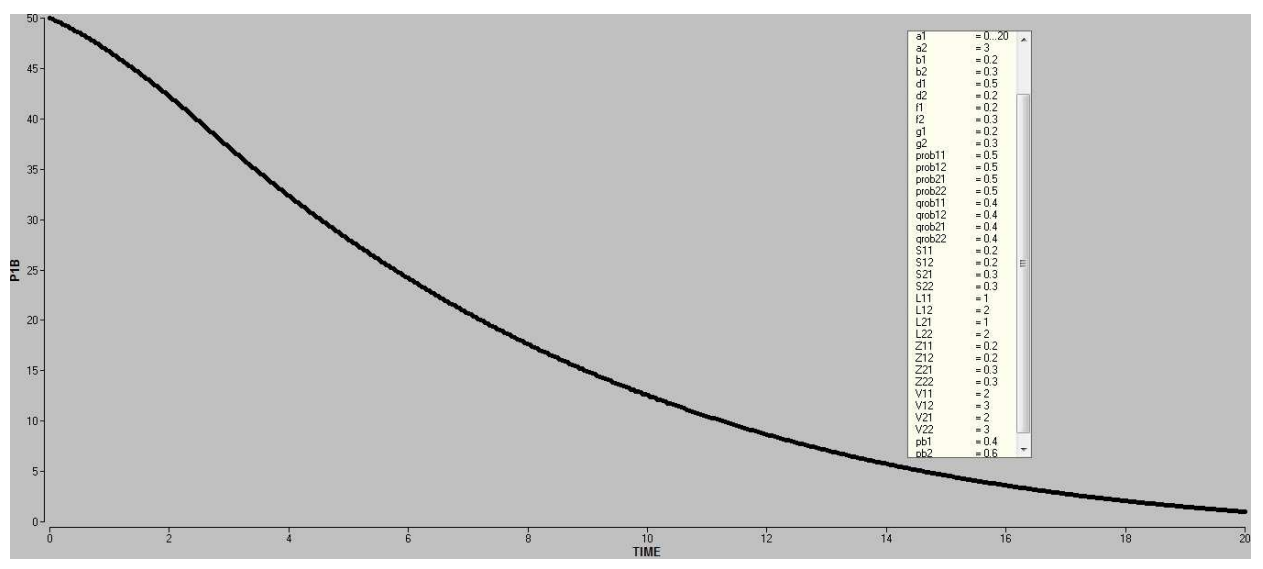

Figure 6: An example where varying the value of $a_{i}$ does not affect $\left[P_{i} B\right]$. 


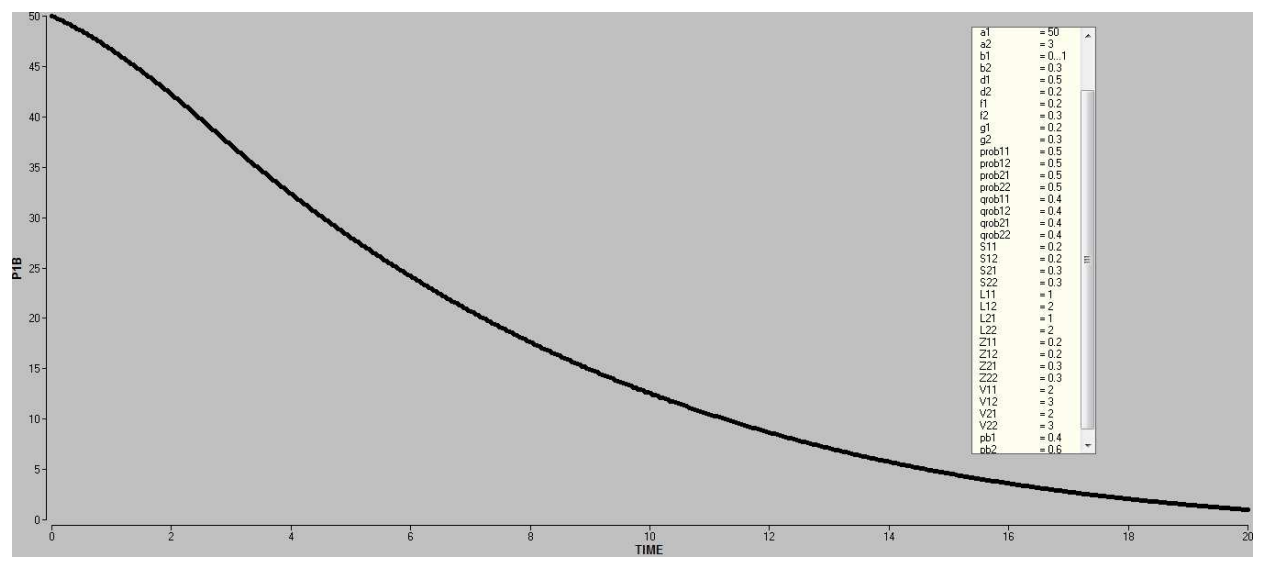

Figure 7: An example where varying the value of $b_{i}$ does not affect $\left[P_{i} B\right]$.

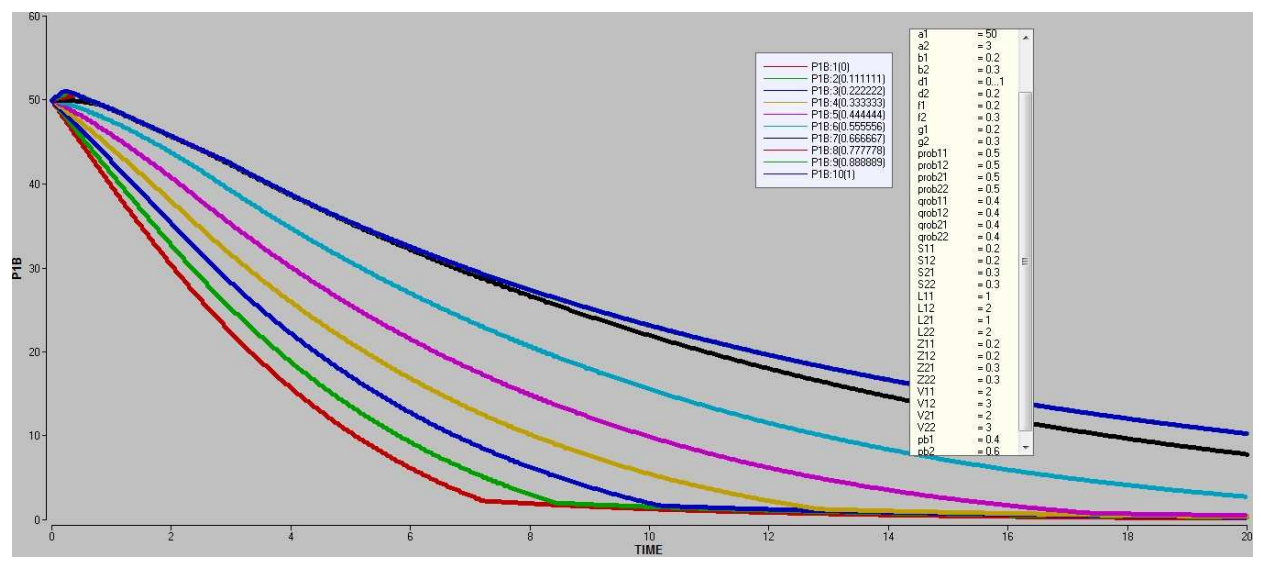

Figure 8: An example where varying the value of $d_{i}$ affects $\left[P_{i} B\right]$.

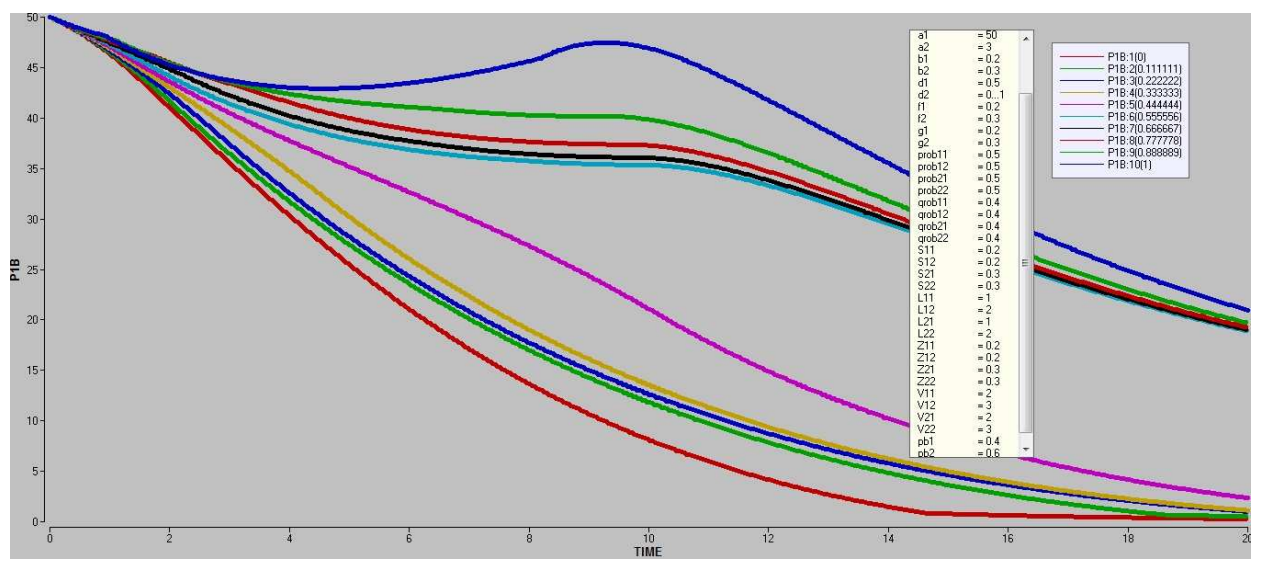

Figure 9: An example where varying the value of $d_{j} j \neq i$ affects $\left[P_{i} B\right]$. 


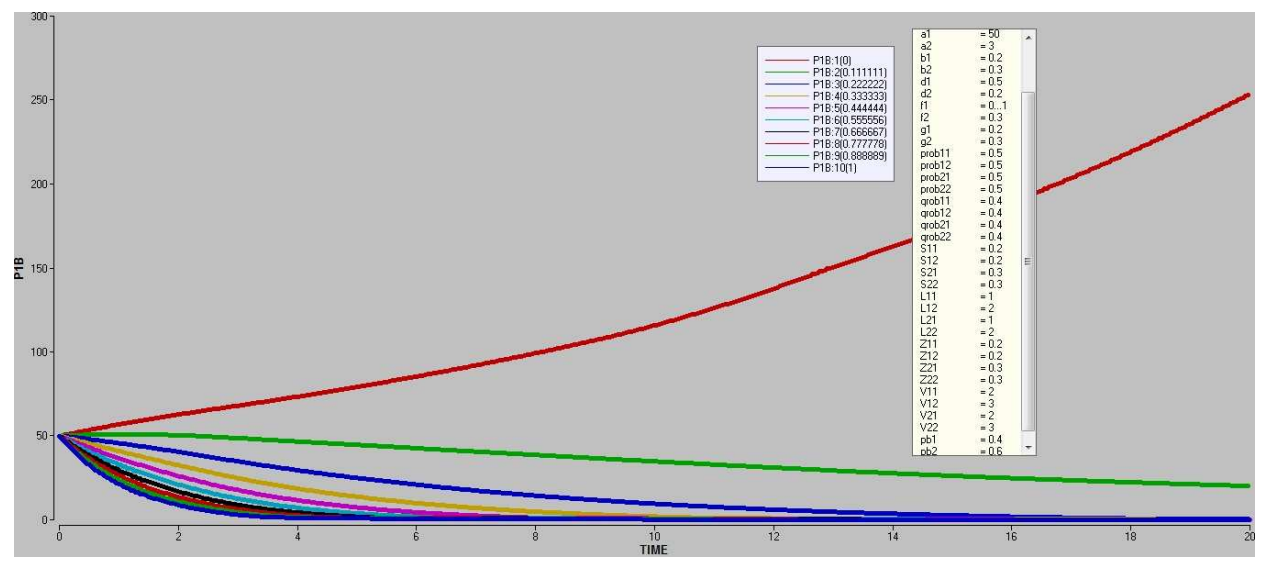

Figure 10: An example where varying the value of $f_{i}$ affects $\left[P_{i} B\right]$.

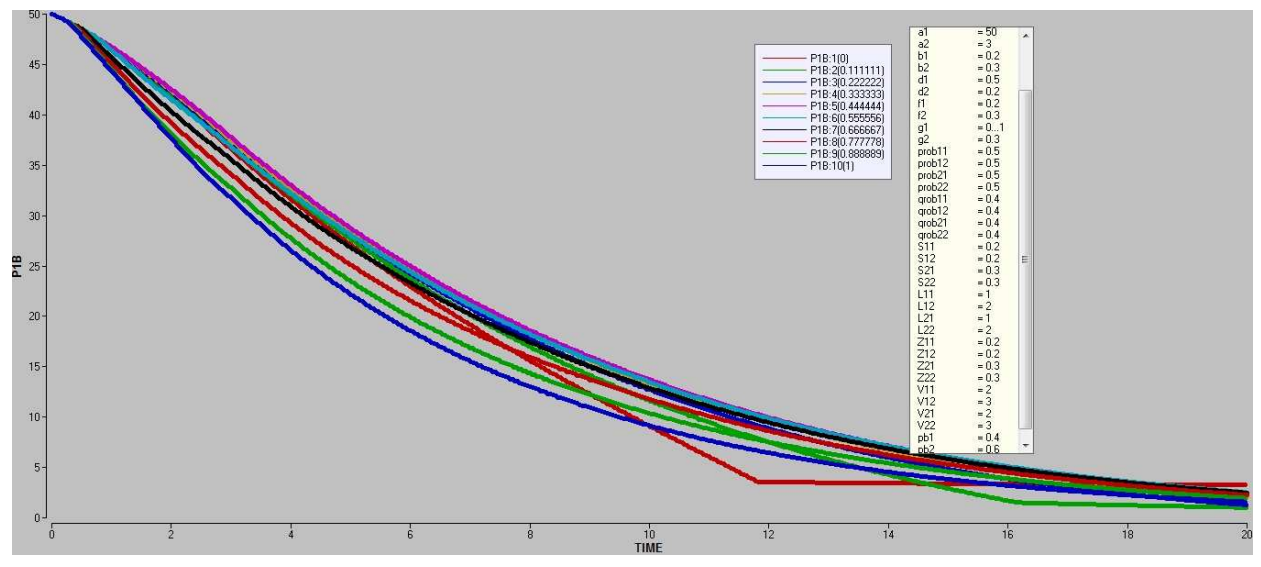

Figure 11: An example where varying the value of $g_{i}$ affects $\left[P_{i} B\right]$.

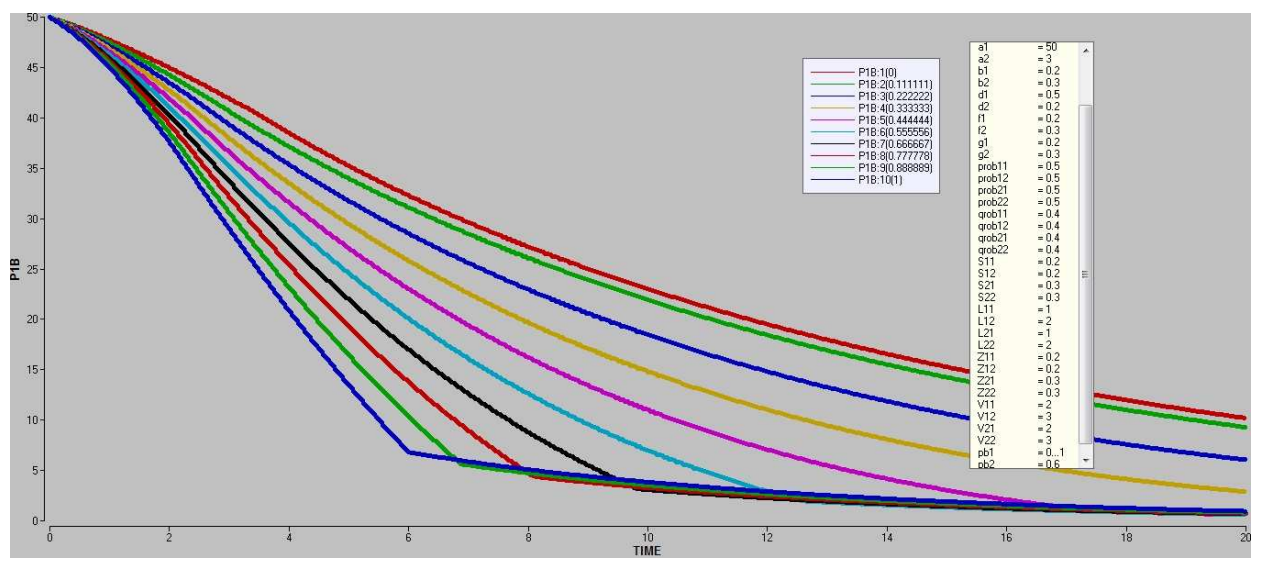

Figure 12: An example where varying the value of $p b_{i}$ affects $\left[P_{i} B\right]$. 


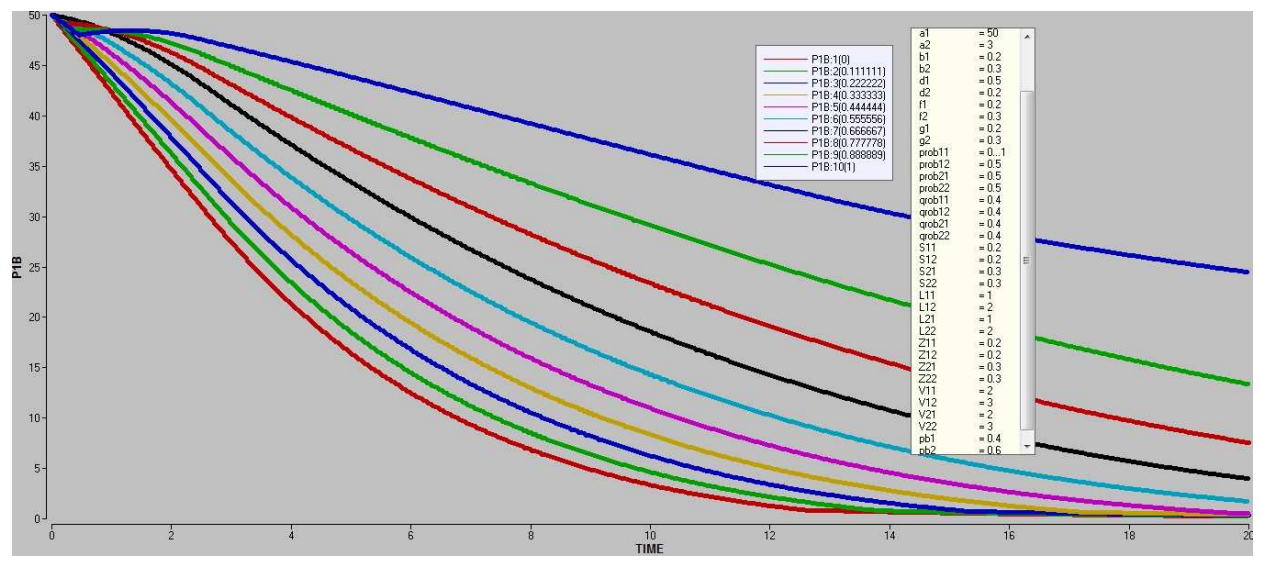

Figure 13: An example where varying the value of $\operatorname{prob}_{i i}$ affects $\left[P_{i} B\right]$.

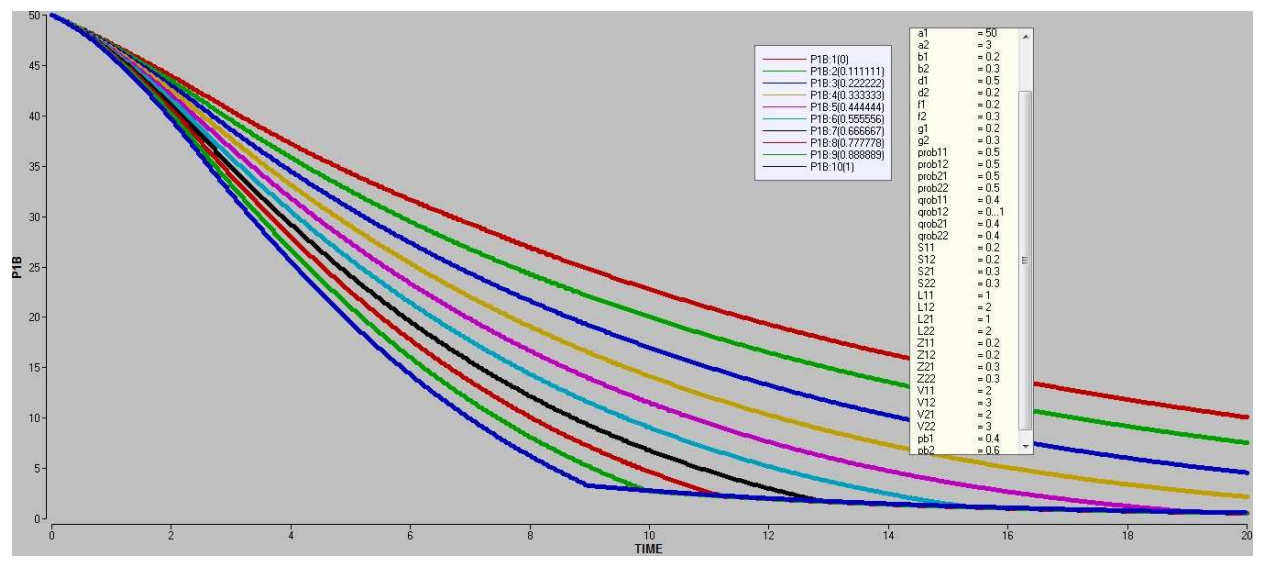

Figure 14: An example where varying the value of $q r o b_{i j} j \neq i$ affects $\left[P_{i} B\right]$.

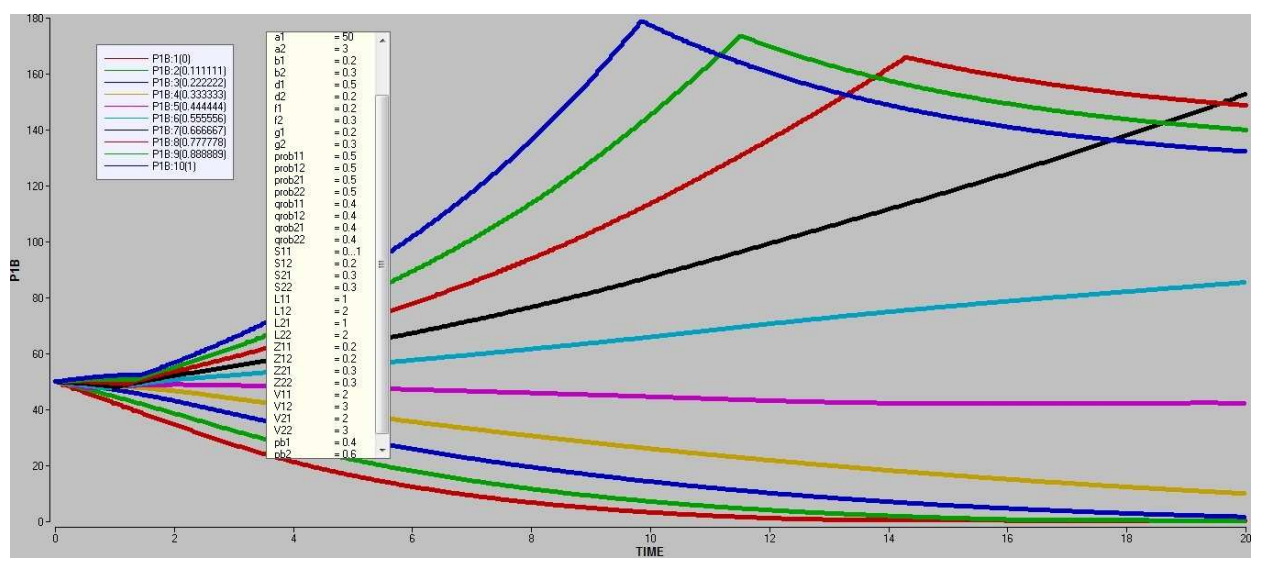

Figure 15: An example where varying the value of $S_{i i}$ affects $\left[P_{i} B\right]$. 